\title{
EXPERIMENTAL STUDY OF HEAT TRANSFER AND PRESSURE LOSS IN CHANNELS WITH MINIATURE V RIB-DIMPLE HYBRID STRUCTURE
}

\author{
Yu Rao and Peng Zhang. \\ Institute of Turbomachinery, School of Mechanical Engineering, \\ Shanghai Jiao Tong University, Shanghai, 200240, P.R. China, \\ E-mail: yurao@sjtu.edu.cn
}

\begin{abstract}
In order to increase the thermal efficiency, gas turbines are designed to operate at higher temperature, which requires a highly efficient cooling structure. The dimples and ribs are effective structures to enhance the convective heat transfer in the cooling channels, which are commonly used in the gas turbine blade internal cooling and combustion chamber cooling. Despite a large number of the literature about the study of the heat transfer performance of the dimples or ribs respectively, few studies dealt with the flow and heat transfer characteristics of a hybrid structure with the ribs and dimples. In the present paper, a novel hybrid cooling structure with miniature Vshaped ribs and dimples is presented, and the flow structure, pressure loss and heat transfer characteristics of a cooling channel with the rib-dimple hybrid structure are investigated. The heat transfer characteristics are obtained using a transient liquid crystal thermography technique. The rib-dimples with three different rib heights of $0.6 \mathrm{~mm}, 1.0 \mathrm{~mm}$ and $1.5 \mathrm{~mm}$ and with the dimple depth of $4 \mathrm{~mm}$ are studied in rectangular channels within the Reynolds number range from 10,000 to 60,000. The experimental study showed that the Nusselt numbers of the channels with the rib-dimples are significantly higher than those of the channel with pure dimples. The friction factor increases rapidly with the height of $\mathrm{V}$ ribs. The $\mathrm{V}$ ribs increase the flow disturbance in the front half of the dimples, leading to an increase of the flow turbulent kinetic energy, increasing the flow mixing dramatically, and breaking out the flow recirculation zone in the front half of the dimples. Therefore the $\mathrm{V}$ rib-dimple hybrid structure on the cooling channel surface provides much larger heat transfer enhancement capability than the conventional dimples.
\end{abstract}

\section{INTRODUCTION}

Increasing the turbine inlet gas temperature is an important measure to improve the efficiency of gas turbines. With the increasement of turbine entry temperature, there is an urgent requirement for more efficient cooling technique for turbine blade. Dimples on the wall is an effective heat transfer enhancement method for gas turbine blade internal cooling.

In the cooling channel, the dimples at the wall provide a significant heat transfer enhancement by producing strong vortex flow near the wall [1-6]. Afanasyev [1] investigated the heat transfer and flow resistance characteristics of spherical dimples. Their studies showed that the heat transfer performance of the spherical dimple is $30 \%-40 \%$ higher than the smooth channels, without any appreciable pressure loss in the turbulent flow. Chyu, et al. [2] studied the heat transfer distribution and flow resistance of teardrop and spherical dimples by using transient liquid crystal experiments. They found that the dimple vortex generators can increase the heat transfer enhancement by increasing turbulence of the flow. Schukin [3] applied the dimples to the turbines cooling, and measured the average heat transfer coefficient. Park, et al. [4] used CFD to simulate the internal flow field and the local heat transfer intensity field of the cooling channel with the dimple vortex generator arranged on the wall, and the results show that the dimples produce strong and orderly vortices, and there is a flow re-attachment in the near-wall region. Rao, et al. [6] conducted both experimental and numerical studies of dimpled channels, and it is indicated that the dimpled surface shows non-uniform heat transfer, and the upstream half of the dimpled surface has much lower heat transfer due to the stationary vortex flow existing there.

Maurer, et al. [7] studied the heat transfer and pressure loss of $\mathrm{V}$ rib array in different Reynolds number through numerical simulation and experimental work. The study showed that the ratio of rib height to width are important parameters that affect the performance of heat transfer and flow resistance. Maurer, et al. [8] studied the V-shaped rib heat transfer characteristics, and showed that the $\mathrm{V}$ rib induced downwashing vortex flow and produced significant heat transfer enhancement in the downstream region. Han, et al. [9] studied the effect of ribs with broken and continuous arrangement on heat transfer and the flow resistance. They found that the broken $\mathrm{V}$ ribs can produce stronger vortices which have a better Heat transfer performance

In the present study, one novel hybrid cooling structure, a combination of the miniature V-ribs and the dimples, is presented. One miniature $\mathrm{V}$-shaped rib is arranged upstream each dimple, with the idea of the $\mathrm{V}$-shaped ribs producing the downwashing vortex to break out the stationary vortex in the dimples, and improving the heat transfer in the upstream half of the dimples, and therefore obtaining a more uniform heat transfer in the dimples. In the present paper, detailed heat transfer characteristics on the surface with the $\mathrm{V}$ rib-dimple hybrid structure are studied by using the transient liquid crystal thermography technique.

\section{NOMENCLATURE}




$\begin{array}{lll}D_{h} & {[\mathrm{~mm}]} & \text { Cannel hydraulic diameter of the rectangular channel } \\ F & {[-]} & \text { Pressure loss coefficient } \\ f_{0} & {[-]} & \text { Pressure loss coefficient based on Blasius formula } \\ H & {\left[\mathrm{~W} / \mathrm{m}^{2} \mathrm{k}\right]} & \text { Heat transfer coefficient } \\ L & {[\mathrm{~mm}]} & \text { Length of heated surface } \\ N u & {[-]} & \text { Local Nusselt number } \\ N u_{0} & {[-]} & \text { Local Nusselt number based on Dittus-Boelter formula } \\ P f & {[-]} & \text { Overall thermal performance parameter } \\ P r & {[-]} & \text { Prandtl number } \\ R e & {[-]} & \text { Reynolds number } \\ T_{W} & {[\mathrm{~K}]} & \text { Wall temperature } \\ T r & {[\mathrm{~K}]} & \text { Airflow bulk-temperature } \\ T i & {[\mathrm{~K}]} & \text { Initial wall temperature } \\ x & {[\mathrm{~mm}]} & \text { Cartesian axis direction } \\ \rho & {\left[\mathrm{kg} / \mathrm{m}^{3}\right]} & \text { Density } \\ k & {[\mathrm{~W} / \mathrm{mK}]} & \text { Thermal conductivity }\end{array}$

\section{EXPERIMENTAL SETUP}

Figure 1 is a schematic diagram of the experimental system for the pressure loss and heat transfer measurements for the channels with V rib-dimple hybrid structure. This experimental setup consists of a variable-speed blower, a settling chamber, a Ventruri flowmeter, a differential pressure transducer, a NI Labview data acquisition system and a test section. The air is drawn into the wind tunnel by the blower and the air mass flow rate is measured by the Venturi tube flow meter. The screen heater is connected with a high-power DC power supply. The airflow can be quickly heated to about $50{ }^{\circ} \mathrm{C}$ through the mesh heater and flows into the test section. The entry boundary layer flow should have a low inlet turbulence intensity and a fully turbulent flow profile, which is typical of most internal flow heat transfer studies [6].

The test plate with $\mathrm{V}$ rib-dimple arrays mounted on the surface is inserted into the test channel from below, and a 15 mm-thick, plexiglass-made support plate is used to support the test plate from the bottom by being tightly bolted with the test channel. A $15 \mathrm{~mm}$-thick plexiglass cover plate is above the test section and the camera is placed at the top of the cover plexiglass to collect data. Immediately upstream and downstream the test plate, there are pressure taps installed in the top wall of the test channel to measure the pressure drop across the $\mathrm{V}$ rib-dimple channel. The mixed-mean temperatures of the air inlet and outlet test section are measured by using two and four calibrated Type- $K$ thermocouples respectively which are inserted into about half of the channel height. Seven K-type thermocouples are placed on the back of the copper plate to obtain the average temperature of the plate.

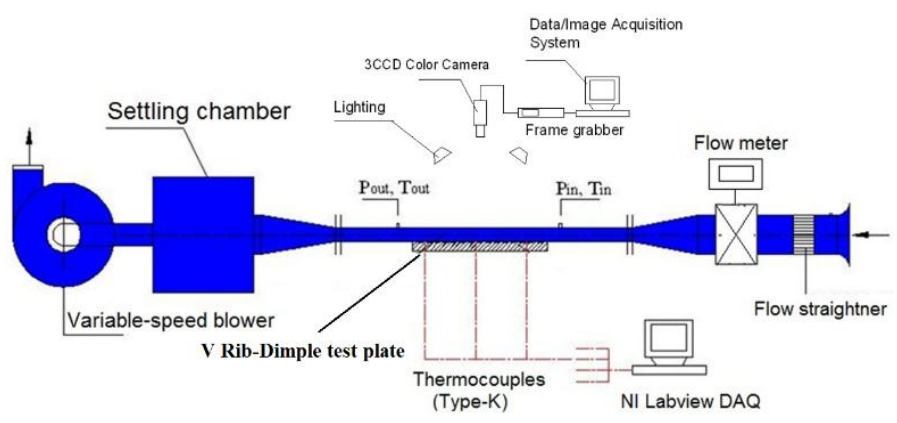

Figure 1 Schematic diagram of the experimental system
Figure 2 is a schematic of the $\mathrm{V}$ rib-dimple test plate. Heights of the V ribs are $1.5 \mathrm{~mm}, 1.0 \mathrm{~mm}$ and $0.6 \mathrm{~mm}$ respectively. The print diameter of the dimples is $20 \mathrm{~mm}$ and the depth is $4 \mathrm{~mm}$. The streamwise spacing $P l=21.6 \mathrm{~mm}$ and the spanwise spacing $P 2=25 \mathrm{~mm}$.

Table 1 The Parameters of test section

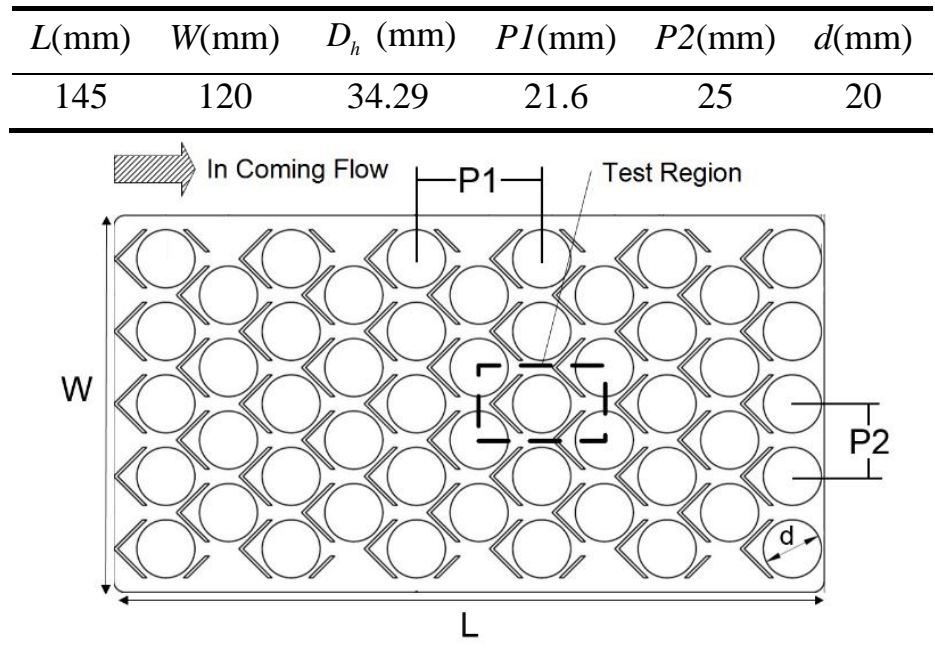

Figure 2 Schematic of V rib-dimples

\section{TRANSIENT TESTING TECHNIQUE DATA REDUCTION}

Figure 3. shows schematic diagram of transient liquid crystal thermography. The black painting was first sprayed directly onto the test plate to improve TLC color visibility for the image acquisition and then covered with a coating of TLC. The supplied TLC has a nominal red start temperature of $35.1^{\circ} \mathrm{C}$ and a bandwidth of $1{ }^{\circ} \mathrm{C}$. The TLC color changing process is recorded using a CCD video camera with a frame rate of 25 frames per second. When a hot air stream flows through the surface with the transient liquid crystal, and the color changes with the increase of temperature. According to the relationship between temperature and color information (Hue values), the temperature information of measured surface at different time can be obtained, then the heat transfer coefficient $h$ is calculated according to the semi-infinite plate heat conduction model. [10-11]. The TLC was carefully calibrated in-situ on the present test section prior to the experiments with the same fluorescent lighting and viewing conditions. The TLC calibration unit consisted of a copper block, thermocouples and an electric heater.

The heat transfer coefficient at each point of the plate surface can be derived from the one-dimensional heat conduction equation of the semi-infinite plate. The onedimensional transient heat transfer model of semi-infinite plate is:

$$
k \frac{\partial^{2} T}{\partial^{2} x^{2}}=\rho c \frac{\partial T}{\partial t}
$$




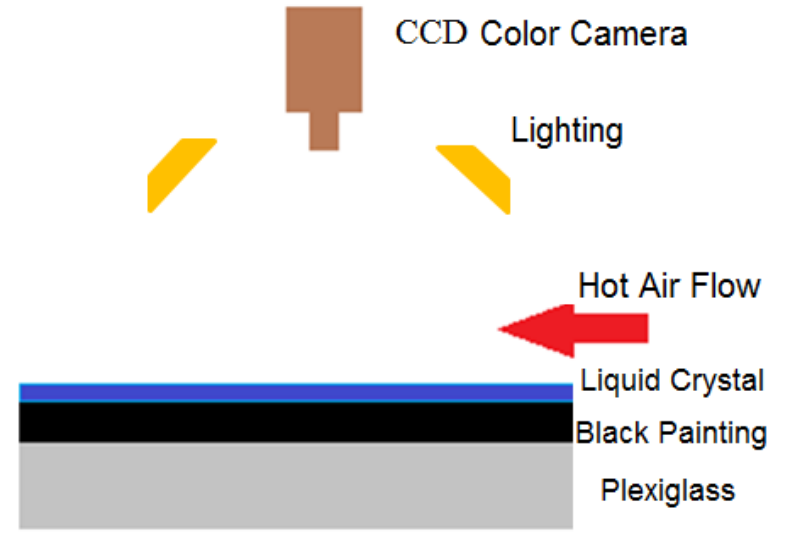

Figure 3 Schematic of transient liquid crystal thermography

Boundary conditions are:

$$
\begin{aligned}
& t=0, T(x, 0)=T_{i} \\
& x=0,-k \frac{\partial T}{\partial x}=h\left(T_{r}-T_{w}\right) \\
& x \rightarrow \infty, T(x, t)=T_{i}
\end{aligned}
$$

$T_{i}$ is the initial temperature of the plexiglass.

$T_{w}$ is the temperature of the plexiglass.

$T_{r}$ is the temperature of the airflow.

According to Duhamel principle and the superposition principle the equation can be simplified as:

$T_{w}-T_{i}=\sum_{j=1}^{N}\left[1-\exp \left(\frac{h^{2} \alpha\left(t-t_{j}\right)}{k^{2}}\right) \operatorname{erfc}\left(\frac{h \sqrt{\alpha\left(t-t_{j}\right)}}{k}\right)\right]\left(T_{r, j}-T_{r, j-1}\right)$

Where $T_{r, j}$, is the airflow bulk-temperature at one specific time $\mathrm{t}_{\mathrm{j}}, T_{w}$ is the measured wall temperature by the time $\mathrm{t}$ (indicated by the color change of the liquid crystals), and $T_{i}$ is the initial wall temperature

The Nusselt number is defined as:

$$
N u=\frac{h D_{h}}{k}
$$

The friction coefficient $\mathrm{f}$ is defined as:

$$
f=\frac{2 \Delta p D_{h}}{\rho U^{2} l}
$$

The Nusselt number of the smooth channel uses the DittusBoelter formula:

$$
N u_{0}=0.023 \operatorname{Re}^{0.8} \operatorname{Pr}^{0.4}
$$

The friction coefficient was calculated using the Blasius formula [12]:

$$
f_{0}=0.316 \operatorname{Re}^{-0.25}
$$

The Reynolds analogy factor is defined as below [13]:

$$
R A F=\frac{N u / N u_{0}}{\left(f / f_{0}\right)}
$$

\section{UNCERTAINTY ANALYSIS}

In this paper, error analysis is performed by using Kline and McClintock's [14] uncertainty methods The accuracy of thermocouple, thermo liquid crystal calibration and measurement time will affect the Nusselt number.

The $\mathrm{K}$ type thermocouple used in this experiment is produced by Omega. The thermocouple has a accuracy of less than $\pm 0.2{ }^{\circ} \mathrm{C}$ after calibrating in a constant temperature water tank; The working temperature of the liquid crystal (SPN$100 / \mathrm{R} 35 \mathrm{C} 1 \mathrm{~W}$ ) is about $35^{\circ} \mathrm{C}$, with a color band of $1{ }^{\circ} \mathrm{C}$ and the calibration accuracy is $\pm 0.2{ }^{\circ} \mathrm{C}$; The air's thermophysical parameter varies with the temperature, which cause an error about $\pm 2 \%$. The processing error of the plexiglass is about $\pm 0.1 \%$. The error of the Plexiglass thermal conductivity variation due to temperature changing is about $4 \%$. In summary, the maximum error of the friction factor obtained by the experiment is $\pm 5 \%$, while the maximum error of the Nusselt number is less than $\pm 8.5 \%$.

\section{EXPERIMENTAL RESULTS AND ANALYSIS Local heat transfer characteristics}

Figure 4 show a distribution of the $\mathrm{Nu} / \mathrm{Nu}_{0}$ contour of the $\mathrm{V}$ rib-dimple channel with rib heights of $0.6 \mathrm{~mm}, 1.0 \mathrm{~mm}$ and 1.5 mm respectively, and Figure 5 shows the $\mathrm{Nu} / \mathrm{Nu}_{0}$ contour of the conventional dimpled channel at the Reynolds number of $10,000,36,700,50,500$. Looking at the $\mathrm{V}$ ribs with a height of $0.6 \mathrm{~mm}$ at $\mathrm{Re}=10,000$ (Figure 4(a)), it is similar to the spherical dimples, the upstream half of the dimpled wall shows low heat transfer owing to the flow separation and recirculation and the convective heat transfer is distinctively enhanced in the downstream half of the dimpled wall and in the downstream area of the dimples, particularly near the rear rim of the dimples, due to the flow attachment and upwashing flow. However, the front plate region of the dimples and the middle and both sides rim of the dimpled wall with $\mathrm{V}$ ribs have a higher heat transfer, which is different from that of spherical dimples. When air flow passes over the $\mathrm{V}$ ribs causing a flow separation, then the air flow attaches on the front plate region of the dimples, which results in thin thermal boundary layer to enhance heat transfer. Meanwhile the reattachment causes a downwash velocity which keeps the air flow traveling towards to the middle of the dimples leads to a high heat transfer performance and reduces the recirculation zone of upstream half of the dimple. the creation of the gap in the leg extremities of V-rib releases the secondary flow and therefore increases the velocity of flow through the gap [15]. The higher-velocity jets are released through the gaps with high turbulence which enhances heat transfer of the both edges rim of the dimples. There is little difference in the local heat transfer distribution between $\mathrm{Re}=10,000$ and higher Reynolds numbers $(\mathrm{Re}=36,700$, $\mathrm{Re}=50,500)$. The area of high local heat transfer in the middle of dimple at $\mathrm{Re}=10000$ is much smaller than that of $\mathrm{Re}=36,700$ and $\mathrm{Re}=50,500$ (Figure 4(d) and Figure 4(g)). This is caused by flow reattachment point moving towards dimple with the velocity increasing, which leads to more air flow reattaches directly on the upstream half of the dimples, thereby enhancing heat transfer.

All the $\mathrm{V}$ rib-dimples show higher and more uniform heat transfer than the pure dimples. The heat transfer patterns change with the ri bs height increasing. The high heat transfer performance areas at the middle of the dimple spread towards 
to the front half of the dimple with the $\mathrm{V}$ ribs heights increasing. The higher rib height causes reattachment point towards dimple leading to more air flow reattaching directly on the upstream half of the dimples which results in the higher heat transfer on middle and lower heat transfer on both edges. The highest heat transfer performance region is at the rear rim of dimpled wall for $0.6 \mathrm{~mm}$ rib heights, at the front region of the dimples for
$1.0 \mathrm{~mm}$ and at the front region of the dimples and rear rim of dimpled wall for $1.5 \mathrm{~mm}$. Accordingly, the different reattachment point due to the diverse $\mathrm{V}$ rib height can influence the flow structure thus changing the highest heat transfer performance region.

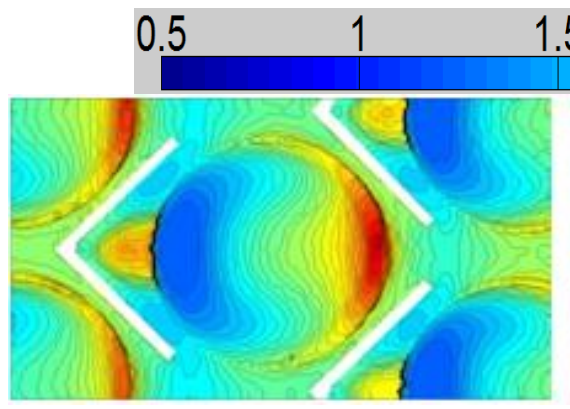

(a)

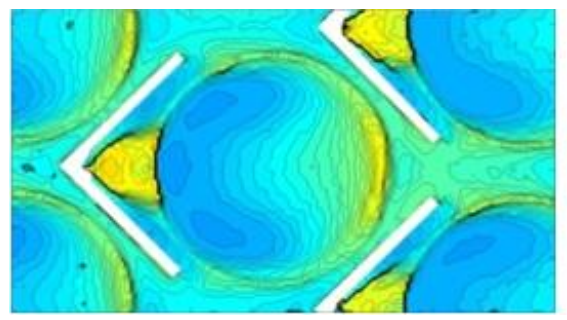

(d)

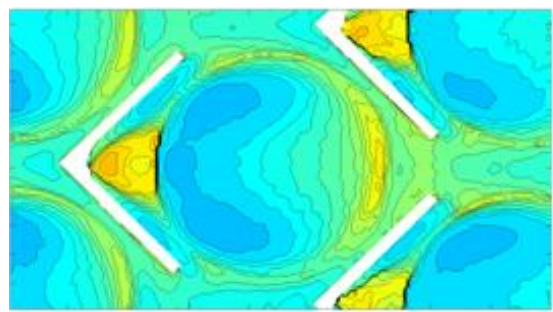

(g)

V rib height: $0.6 \mathrm{~mm}$

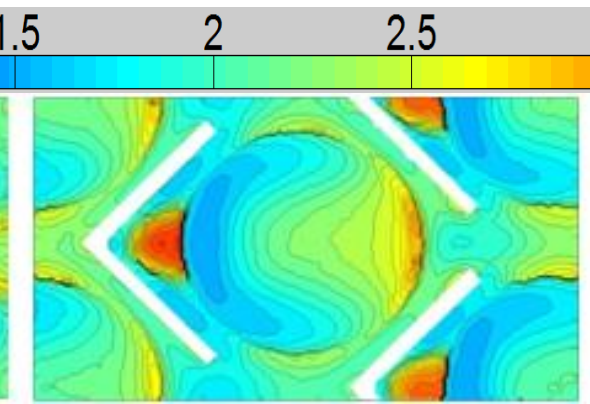

(b)

$R e=10,000$

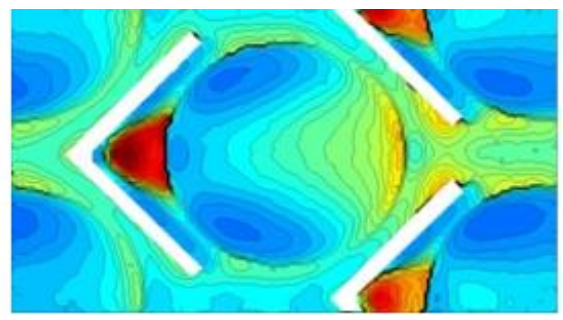

(e)

$\operatorname{Re}=36,700$

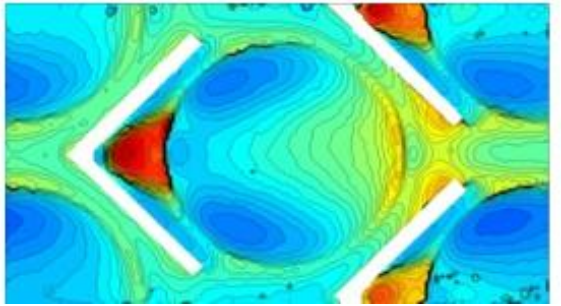

(h)

$R e=50,500$

V rib height: $1.0 \mathrm{~mm}$

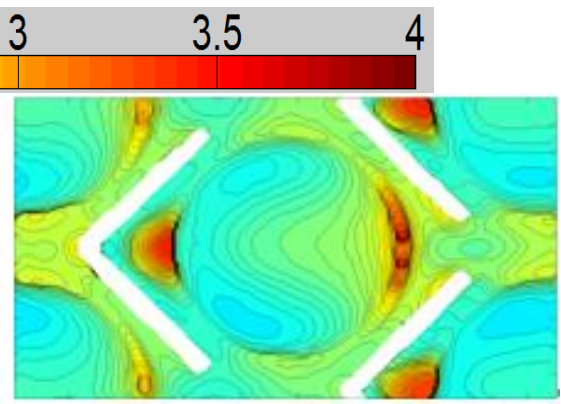

(c)

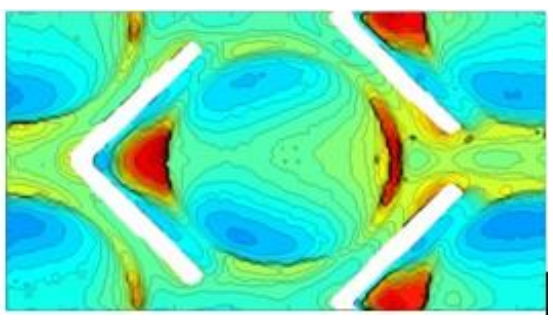

(f)

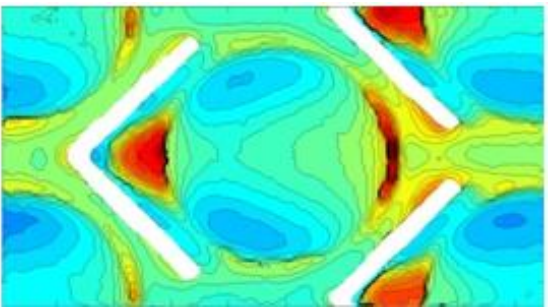

(i)

Figure 4 Comparisons of $\mathrm{Nu} / \mathrm{Nu} 0$ of $\mathrm{V}$ rib-dimple channels with different rib heights

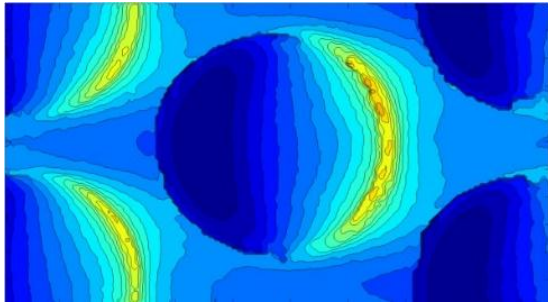

(a) $\mathrm{Re}=10,000$

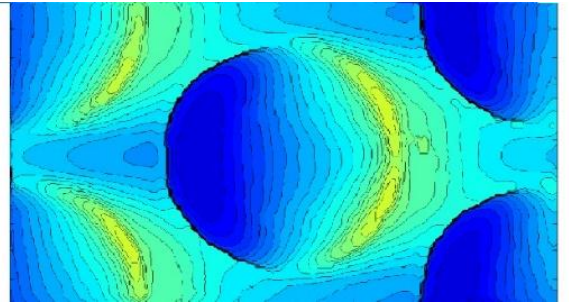

(b) $\mathrm{Re}=36,700$

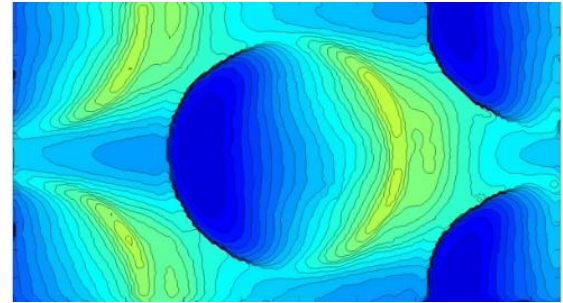

(c) $\operatorname{Re}=50,500$

Figure $5 \mathrm{Nu} / \mathrm{Nu}_{0}$ contour of the dimpled channel (the legend is the same with Fig.4) 


\section{Average heat transfer and friction factors}

Figure 6 shows the $\mathrm{Nu}$ and $\mathrm{Nu} / \mathrm{Nu}_{0}$ diagrams for the $\mathrm{V}$ ribdimple channels with different rib heights within the Reynolds number range of 10,000-60,000. The experiment shows that the $\mathrm{V}$ rib-dimple channels with different rib heights have much higher heat transfer performance than the smooth channel and the counterpart dimple channel. With the Reynolds number increasing, the $\mathrm{Nu} / \mathrm{Nu}_{0}$ values of the $0.6 \mathrm{~mm}$ and $1.0 \mathrm{~mm} \mathrm{~V}$ ribdimple keep almost constant values, which are about 2.0 and 2.1 respectively. However the $\mathrm{Nu} / \mathrm{Nu}_{0}$ values of the $1.5 \mathrm{~mm} \mathrm{~V}$ rib-dimple keep almost constant values of about 2.3 firstly and then increase appreciably up to about 2.45 , and this is mainly due to the reason that the $\mathrm{V}$ rib with the $1.5 \mathrm{~mm}$ height dominates the heat transfer enhancement capability at the surface under stronger turbulent flow conditions, which increases with the Reynolds numbers and is also shown in Ref. [16]. Obviously the V rib-dimple channel provides higher heat transfer enhancement than the dimpled channel by about $24 \%$ $53 \%$.

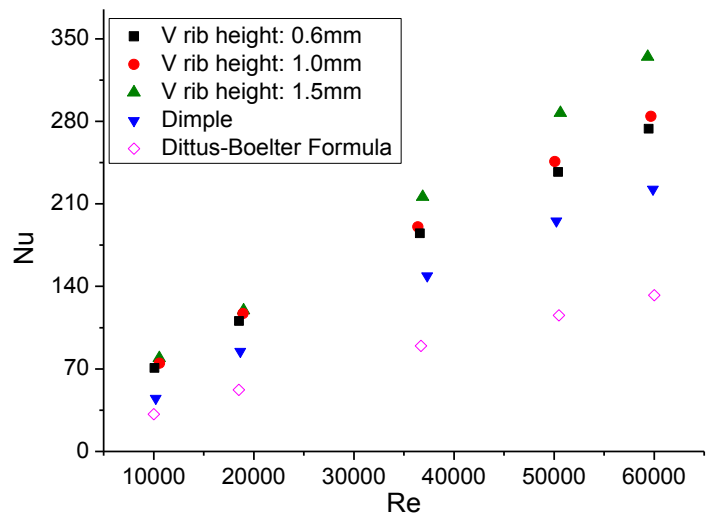

(a)

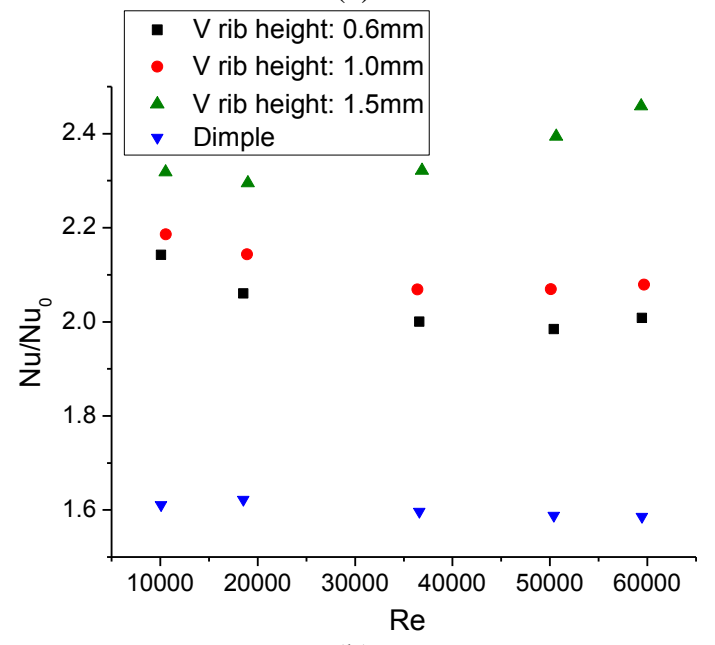

(b)

Figure $6 \mathrm{Nu}$ and $\mathrm{Nu} / \mathrm{Nu}_{0}$ of the $\mathrm{V}$ rib-dimple channels

Figure 7 shows the $\mathrm{v}$ rib-dimple channel friction factor $f$ and $f / f_{0}$ for different rib heights. The experiment shows that the pressure loss of the V-rib -dimple is larger than that of the spherical dimple and the smooth flat in the range of experimental Reynolds number. The pressure loss of the $\mathrm{V}$ rib- dimple increase with the rib height increasing. The pressure loss of $0.6 \mathrm{~mm}$ and $1.0 \mathrm{~mm}$ rib height are close, which is about 2.24-3.22 times than that of the smooth channel.

Figure 8 shows Reynolds analogy factors of the channel with $\mathrm{V}$ rib-dimples at different Reynolds number. With the increase of Reynolds number, the Reynolds analogy factors of the $\mathrm{V}$ rib-dimple of $0.6 \mathrm{~mm}, 1.0 \mathrm{~mm}$ and $1.5 \mathrm{~mm}$ decreases gradually. The dimple shows the highest Reynolds analogy factors while the Reynolds analogy factors of $1.5 \mathrm{~mm} \mathrm{~V} \mathrm{rib-}$ dimple is the lowest within all Reynolds number. The Reynolds analogy factors of the $\mathrm{V}$ rib-dimple is $2.0 \%-40.0 \%$ lower than that of the dimple and the Reynolds analogy factors of $1.5 \mathrm{~mm}$ $\mathrm{V}$ rib-dimple is $21.1 \%-41.2 \%$ lower than that of $0.6 \mathrm{~mm}$ and $1.0 \mathrm{~mm} \mathrm{~V}$ rib-dimple.

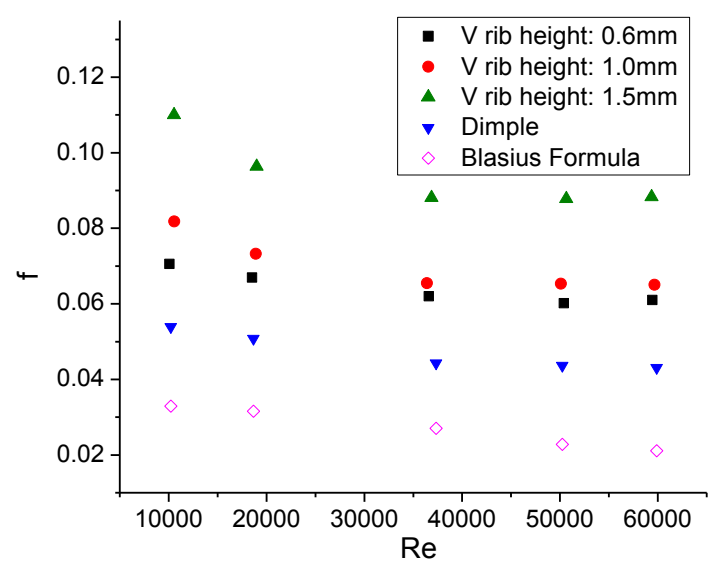

(a)

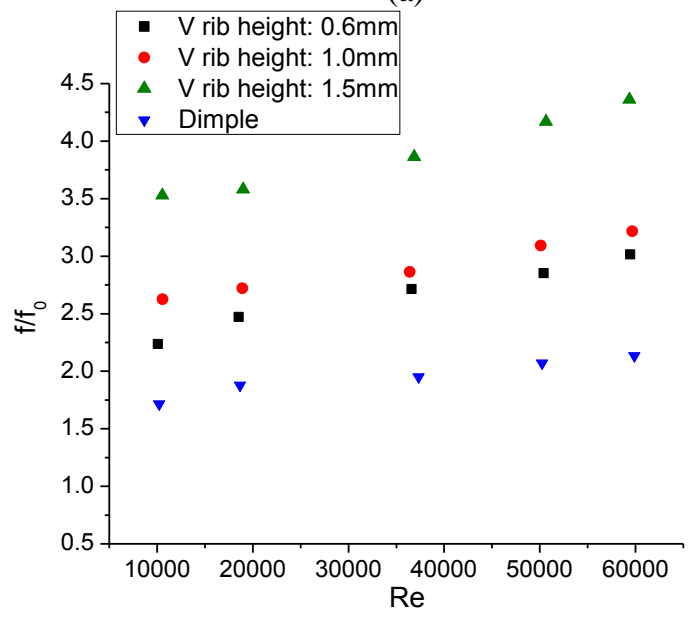

(b)

Figure. $7 f$ and $f / f_{0}$ of the V rib-dimple channels 


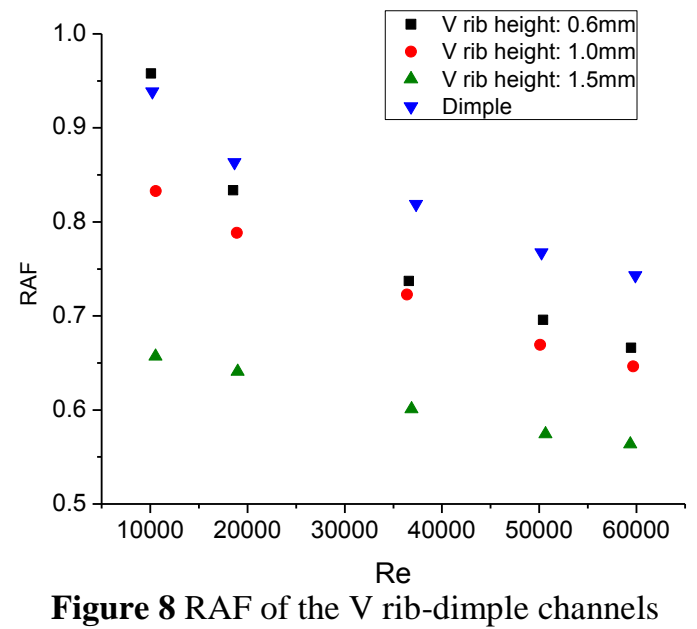

\section{CONCLUSION}

This paper presented an experimental study on the heat transfer and pressure loss performance of a turbulent flow over surfaces with micro V rib-dimple hybrid structures. Different V rib heights of $0.6 \mathrm{~mm}, 1.0 \mathrm{~mm}$ and $1.5 \mathrm{~mm}$ are studied. The detailed heat transfer characteristics on the channel surface with the $\mathrm{V}$ rib-dimples have been obtained and compared with each other for the Reynolds number range of 10,000-60,000.

It is found that the heat transfer and pressure loss of the channel with micro $\mathrm{V}$ rib-dimples are significantly influenced by the rib heights. The channel with $\mathrm{V}$ rib-dimples has higher heat transfer performance than the smooth channel and the channel with pure dimples. The heat transfer and friction factor of the channel with the $\mathrm{V}$ rib-dimples are respectively about 2.0-2.5 times and 2.2-4.3 times those of smooth channel. Compared with the conventional dimpled channel, the heat transfer performance of the the $\mathrm{V}$ rib-dimple channels is about 24.0\%-53.1\% higher, and the friction factors are about 30.3\%$112.5 \%$ higher. With the increase of the Reynolds number, the Reynolds analogy factors of the $\mathrm{V}$ rib-dimples decreases gradually, and the $\mathrm{V}$ rib-dimple with a lower rib height shows higher Reynolds analogy factors.

\section{REFERENCES}

[1] Afanasyev V N, Chudnovsky Y P, Leontiev A I, et al. Turbulent flow friction and heat transfer characteristics for spherical cavities on a flat plate. Experimental Thermal and Fluid Science, Vol. 7, 1993, pp. 1-8.

[2] Chyu M K, Yu Y, Ding H. Heat transfer enhancement in rectangular channels with concavities. Journal of Enhanced Heat Transfer, Vol. 6, 1999 pp. 429-439.

[3] Schukin A V, Kozlov A P, Agachev R S. Study and application of hemispherical cavities for surface heat transfer augmentation. ASME paper, 95-GT-59, 1995.

[4] Park J, Desam P R, Ligrani P M. Numerical predictions off low structure above a dimpled surface in a channel. Numerical Heat Transfer, Part A: Applications, Vol. 45, 2004, pp. 1-20.

[5] Won S Y, Ligrani P M. Numerical predictions off low structure and local Nusselt number ratios along and above dimpled surfaces with different dimple depths in a channel. Numerical Heat Transfer, Part A: Applications, Vol. 46, 2004, pp. 549-570.

[6] Rao, Y, Feng, Y, Li, B, Weigand, B, Experimental and numerical study of heat transfer and flow friction in channels with dimples of different shapes, ASME Journal of Heat Transfer, Vol. 137, pp. 031901, 2015.

[7] Maurer M, von Wolfersdorf J, Gritsch M. An experimental and numerical study of heat transfer and pressure losses of V-and Wshaped ribs at high Reynolds numbers. ASME paper, GT200727167, 2007.

[8] Maurer M, Wolfersdorf J V, Gritsch M. An experimental and numerical study of heat transfer and pressure loss in a rectangular channel with V-shaped ribs. Journal of Turbomachinery, Vol. 129, 2007, pp. 800-808.

[9] Han J C, Zhang Y M. High performance heat transfer ducts with parallel broken and V-shaped broken ribs. International Journal of Heat and Mass Transfer, Vol. 35, 1992, pp. 513-523.

[10] Wang Z, Ireland P T, Jones T V, et al. A color image processing system for transient liquid crystal heat transfer experiments. Journal of Turbomachinery, Vol. 118, 1996, pp. 421-427.

[11] Ekkad S V, Han J. A transient liquid crystal thermography technique for gas turbine heat transfer measurement. Measurement Science and Technology, Vol. 11, 2000, pp. 957968.

[12] Kays W M, Crawford M E. Convective heat and mass transfer, third edition. USA: McGraw Hill, 1993.

[13] Ligrani P M, Oliveira, M M, Blaskovich, T. Comparison of heat transfer augmentation techniques, AIAA Journal, Vol. 41, pp. 337-362, 2003.

[14] Kline S J, Mcclintock F A. Describing uncertainties in singlesample experiments, Mechanical Engineering, Vol. 75, 1953, pp. 3-8.

[15] Anil K P, J. S. S and Krishna K. Experimental Investigation of Enhanced Heat Transfer and Pressure Drop in a Solar Air Heater Duct With Discretized Broken V-Rib Roughness, Journal of Solar Energy Engineering ,Vol. 137, 2014, pp. 021013.

[16] Hagari, T, Ishida, K, Oda, T, Douura, Y, Kinoshita, Y, Heat transfer and pressure losses of $\mathrm{W}$-shaped small ribs at high Reynolds numbers for combustor liner, Journal of Engineering for Gas Turbine and Power, Vol. 133, 2011, pp. 091901. 